\title{
The Aerodynamic Drag of Parafoils
}

\author{
A. C. Carruthers* and A. Filippone ${ }^{\dagger}$ \\ The University of Manchester \\ Manchester M60 1QD \\ United Kingdom
}

\section{Introduction}

The parafoil is an aerodynamic decelerator that uses the concept of parachute and airfoil aerodynamics. The device has been used as a parachute for high-precision delivering of payload (military and civilian), to recover sounding rockets, for various tethered applications (to carry instrumentation for weather survey, air pollution measurements, radar tracking systems), and as recreational parachuting since the 1970s, as discussed by Nicolaides? and Knapp \& Barton? . All these systems take advantage of the low landing speed and manoeuvrability of the parafoil. The invention is rather old, and it was used as far back as the second World War for stabilisation of supersonic vehicles by H.G. Heinrich, as reported by Meyer? . The essential concept was that in addition to suitable aerodynamic resistance (as the parachute), the parafoil had to provide lift, stability and control, so that it could be used for high precision landing.

The technical literature in the field has addressed aerodynamics, performances, and longitudinal stability, from flight testing, wind tunnel measurements and theoretical models. Relevant publications in the field include the work of Lingard? , who showed

\footnotetext{
${ }^{*}$ Graduate Student, AIAA Student Member

${ }^{\dagger}$ Lecturer, AIAA senior member. Faculty of Engineering and Physical Sciences, Dept. of Mechanical, Aerospace, Civil Engineering. Corresponding author. E-mail: a.filippone@manchester.ac.uk
} 
equations for the lift and drag coefficients from low speed aerodynamic theory, and discussed the effects of wind speed, geometry and size of the parafoil. Other studies address glide ratio and rate of descent under various wind conditions, in addition to deployment/inflation mechanisms, warping, turn control, effects of rigging lines, and more. These analyses are not directly applicable. A summary of this literature is given by Matos et al? .

This paper discusses the low speed wind tunnel drag characteristics of parafoils. The devices tested are flexible (but not inflatable) strips of cloth that adjust to the conditions of the incoming wind, and produce relatively large drag forces and little or no lift. Therefore, they are essentially aerodynamic decelerators with spanwise camber increasing with their length, since their edges were tied at a constant distance. Ram pressure builds up on the side of the parafoil facing the free stream and creates considerable drag.

\section{Experimental Set-up}

The parafoils were mounted at two ends on a U-shaped support rod, as shown in Fig. ??. This distance was maintained constant in the experiments described. No rigging lines of any length have been used.

Measurements were taken in a low speed wind tunnel having a working test section of $0.9 \times 0.9 \mathrm{~m}, 3.14 \mathrm{~m}$ long. The reading of the forces was done with a three-component balance. The balance was mounted outside the tunnel wall, mid-way along the working section. A Pitot static probe was placed upstream the model for velocity measurements.

Twelve parafoils of cotton cloth were cut. Three planform areas were used: 0.025 $\mathrm{m}^{2}, 0.05 \mathrm{~m}^{2}$, and $0.075 \mathrm{~m}^{2}$. For each of the planforms four aspect ratios have been considered: $3.3,10,20$, and 30 .

The relevant material properties of the fabric used are as follows: weight $\mathrm{W}=177$ $\mathrm{gr} / \mathrm{m}^{2}$, bending rigidity $0.0642 \mathrm{gr} \mathrm{cm}^{2} / \mathrm{cm}$; bending hysteresis $0.0446 \mathrm{gr} \mathrm{cm} / \mathrm{cm}$. The geometrical characteristics of the parafoils are the same as described previously? 
A $12 \mathrm{~mm}$ diameter U-shaped mounting rod was connected directly to the force balance, Fig. ??. The parafoils were attached to the vertical arms of mounting rod. Rubber O-rings were placed around the mounting rod. A string was used to connect these to the eyelets of the parafoils, so that the fixed edges were pulled taut and tight against the downwind side of the mounting rod. This was manufactured such that the parafoil ends would be held 0.42 metres apart. This was fixed length equal to less than half the wind tunnel's width, to avoid complicated wall effects. In any case, the relevant geometric parameter of the arrangement is the ratio $L / b$ that is a geometric similarity. A greater or longer arm would increase or decrease the Reynolds number, but the effects of this parameter on the results have been found small compared to the aspect ratio and the planform area. Those parafoils not long enough to span this distance had a string pulling the parafoil tight between the vertical struts of the mounting rod.

The velocity range in the wind tunnel test section was between 6 and $18 \mathrm{~m} / \mathrm{s}$, corresponding to Reynolds numbers in the range $1.7 \cdot 10^{5}$ to $5.2 \cdot 10^{5}$. The Reynolds number was calculated by using as a reference length the horizontal arm $b$ of the Usupport rod, Fig. ??. The wind speed was measured using a Pitot static probe. The probe connected to a high-accuracy digital manometer that took readings within 0.01 $\mathrm{mm} \mathrm{H}_{2} \mathrm{O}(0.0981 \mathrm{~Pa})$.

Force measurements were taken using a force balance through which the mounting rod was secured. A voltage-time series was taken for each data point, 2048 samples over 5 seconds, and averaged to give a mean drag measurement. The voltage output was converted to a force measurement through a calibration curve obtained by applying loads at the centre of the force balance. A vibration unit was connected through the balance and used immediately before readings were taken, to reduce sticking of the mounting rod through the force balance collet.

Each parafoil was tested six times over two days. The results were averaged to give drag coefficient $\times$ area results. Error bars were calculated using the maximum and minimum deviation from the average. 


\section{Experimental Difficulties}

During testing it was found that the parafoil having the lowest aspect-ratio and the lowest planform area $\left(A R=3.3, A=0.025 \mathrm{~m}^{2}\right)$ suffered significant vibrations at the upper end of the velocity range. The velocity at which this vibration occurred was sensitive to minor differences in mounting, i.e. the tightness of the string attaching the parafoil to the mounting rod O-rings. Once this severe vibration began, to avoid damage to the force balance we then operated changes in the wind tunnel speed, or stopped the tunnel and loosened the mount.

The highest aspect ratios, particularly parafoils with $A R=30, A=0.05 \mathrm{~m}^{2}$ and $A=0.075 \mathrm{~m}^{2}$, were observed to twist up at the lowest velocities. This required that the parafoils be tested from the highest velocity down to the lowest velocity, rather than the other way around.

When the parafoils twisted up, sometimes they could be returned to an untwisted state by lowering or increasing the velocity — thus altering the vibrations suffered by the parafoils.

Once reaching a wind speed of $8 \mathrm{~m} / \mathrm{s}$ and below the tendency for the parafoils to twist occurred again. This was not easily sorted, and the drag measured at these points are therefore highly dependent on the orientation of the parafoil at each time interval.

\section{Results and Discussion}

The drag data for the parafoils are presented in terms of the product $C_{D} A(\operatorname{drag} \times$ reference surface): $C_{D} A$ is the ratio between the drag force and the dynamic pressure $q=\rho U^{2} / 2$, a quantity readily measurable. Our choice was dictated by the fact that a reference area is arbitrary: it can be the wetted area, the planform area, the area offered to the incoming flow. Whilst the first two are variable with the parafoil's geometry, the latter one is more arbitrary, because of forced oscillations that sometimes led to a wrapping and twisting of the parafoils, as discussed above. 
The effects of aspect ratio on the drag is shown in Figs. ??-??, in the whole range of speeds tested, for a given wetted area. Each graph shows refers to a fixed planform area. The effects of aspect ratio are stronger then the effects of increasing wind speed. At each value of the aspect ratio the drag data are concentrated over a narrow range. The average data over this speed range are fitted with an exponential function like $C_{D} A=\exp (a A R+b)$, with the coefficients $a$ and $b$ as shown in the figures. From this result we concluded that low-aspect ratio parafoils are more efficient drag devices.

The result shows that the quantity $C_{D} A$ on the parafoils scales roughly with the planform area. Therefore, by taking as a reference area the planform, the drag coefficient is not dependent on the length of the parafoil, provided the aspect ratio is maintained constant.

We conclude that the same device tied at both ends and facing the wind is as much as 8 times more effective as a decelerator system than the device attached at one end only.

The effects of wind speed on the drag force of the parafoils are shown in Fig. ??, Fig. ??, Fig. ?? and Fig. ?? at fixed aspect ratios, for all the planform areas tested. The error bars for some cases are included. These bars have been eliminated where they were too narrow, in order to keep the charts clear. The data obtained make it clear that the effect of the wind speed is of minor relevance compared to the effect of the planform area. The coefficient $C_{D} A$ is only slightly decreasing with the increasing wind speed and Reynolds number. We note that the data obtained at the larger aspect ratios (20 and 30) are more prone to oscillations, and therefore present a larger error bar. These cases are the ones that tended to warp at the low wind speeds, therefore the drag measurements were highly dependent on the geometry of the warping that occurred at each test. At high speeds the error is due to increasingly violent oscillations, relatively high in amplitude and frequency.

Fig. ?? to Fig. ?? show the effects of wind speed at constant aspect-ratio for parafoils having planform area equal to $0.025,0.050$ and $0.075 \mathrm{~m}^{2}$, respectively. Again, the effects 
of wind speed seem negligible in comparison to the other parameters (aspect-ratio and planform area).

The efficiency of the parafoil as an aerodynamic decelerator can be calculated as the ratio between the aerodynamic drag force and the parafoil's own weight at a given wind speed, $D / W$. Consider the parafoil having the mean planform, $A=0.050 \mathrm{~m}^{2}$ and aspect-ratio $A R=3.3$. Fig. ?? indicates that an average value of the drag force $D \sim 7.5 \mathrm{~N}$ at the average wind speed in the range tested, $12 \mathrm{~m} / \mathrm{s}$. Therefore the ratio sought is $D / W \simeq 850$ - a fairly large number that can be verified for the other cases.

\section{Modes of Oscillation}

The modes of oscillation of the parafoils are dependent on the aspect ratio. At the low aspect ratio, $A R=3.3$, the parafoils are relatively wide, and do not twist at any velocity. These parafoils tend to catch in the flow being pulled taut, and suffer slight flapping motions with increasing velocity. This flapping becomes stronger at the higher wind speeds, but not excessively so, except in the case $A=0.025 \mathrm{~m}^{2}$.

The parafoils of aspect ratio $A R=10$ catch immediately in the air flow, with slight oscillations beginning to appear by $9 \mathrm{~m} / \mathrm{s}$. As the airflow is increased to $17 \mathrm{~m} / \mathrm{s}$, the oscillations become stronger, a fact compounded by the increasing planform area. The parafoils with aspect ratio $A R=20$ had similar oscillation mode, with a low frequency oscillatory movement at wind speed. This was more notable for the larger planform areas.

At higher velocities the oscillations become more pronounced and the centre of the parafoil has a cross-over motion. This motion is caused by each half of the parafoil tending to move in a figure eight (8), as seen in the case of the streamers? . However, as the two sides are linked in the centre, this figure eight tendency is reduced, and the two sides move up and down, one moving up while the other moves down, and a motion similar to a cross is observed with the point at the centre moving least.

The parafoils of aspect ratio $A R=30$ have a tendency to twist at the lowest 
velocities tested and were therefore tested from the highest velocity toward the lowest. At the highest velocity a high-amplitude, high-frequency oscillation is observed, which diminishes in amplitude and frequency as the wind speed is reduced. The parafoils with smallest planform area, $A=0.025 \mathrm{~m}^{2}$ do not have problems with twisting. At a wind speed of about $12 \mathrm{~m} / \mathrm{s}$ there is a twist of the parafoils. This effects does not always occur, but it does in the majority of instances, and can be sorted out by quick alterations in wind speed, then settling back to the required testing point. However, at around $8 \mathrm{~m} / \mathrm{s}$ the twisting occurs again, but for this and lower velocities the parafoil once twisted will not unwrap.

We concluded that there must be a critical value of the ratio between the length of the parafoil and the distance between the ends that creates a twisting and warping behaviour. The length $L / b$ (ratio of the parafoil length to its arm) must be around 2 or below for avoiding oscillations. For values $L / b$ up to 2.5 there may occur a warping and twisting, but this depends on how tight the attachment is. For higher values we cannot guarantee that the parafoil will be free of unwanted oscillations.

For cases where no wrapping or oscillations occur, the shape assumed by the parafoils is approximately a catenary, because the problem is similar to a tension-resistant fibre under constant load (the ram pressure created by the wind).

\section{Conclusions}

Cotton parafoils of all combinations of four aspect ratios and three planform areas were tested over a range of wind speeds. The time averaged data show that:

- Effect of Aspect-ratio. It is the most important parameters affecting the drag of a parafoil. It is proved that the drag decreases exponentially with the increasing aspect-ratio. Higher drag is obtained with comparatively low aspect ratios.

- Effect of Wind Speed. The wind speed is the weakest parameter in terms of 
parafoil drag — all other parameters being the same.

- Effect of Planform Area. The parafoils' drag scales roughly with the planform area. To a doubling of the area it corresponds a doubling of the drag force, all other parameters being constant.

- Decelerator Efficiency. The parafoil is very efficient as an aerodynamic decelerator, particularly when considering its own weight. The drag forces are several thousand times the parafoils' own weight.

- Fluctuations under the wind. Most parafoils caught the wind and did not suffer twisting. The exception to this was the largest aspect-ratio parafoil $(A R=30)$ with planform areas $0.05 \mathrm{~m}^{2}$ and $0.075 \mathrm{~m}^{2}$. The ratio between the arm of the support and the length of the parafoil $L / b$ reaches a critical limit of about 2.5; above this value wrapping is very likely. At values $L / b<2$ the parafoil assumes the shape of a catenary and is stable under the wind loading. Oscillations at the intermediate values of $L / b$ are likely to be related to the tightness of the mount. 


\section{Symbols}

$$
\begin{aligned}
& A=\text { reference area }\left(m^{2}\right) \\
& A R=\text { aspect ratio } \\
& C_{D}=\text { drag coefficient } \\
& D=\text { drag force }(N) \\
& l=\text { reference length }(m) \\
& R e=\text { Reynolds number } \\
& R e_{c}=\text { critical Reynolds number } \\
& u=\text { wind speed }(\mathrm{m} / \mathrm{s}) \\
& W=\text { weight }
\end{aligned}
$$


List of Figures 